\title{
Correction to: Urine Sediment Recognition Method Based on Multi-View Deep Residual Learning in Microscopic Image
}

Xiaohong Zhang ${ }^{1} \cdot$ Liqing Jiang ${ }^{1} \cdot$ Dongxu Yang ${ }^{1} \cdot$ Jinyan Yan ${ }^{1} \cdot$ Xinhong Lu $^{2}$

Published online: 12 March 2020

(C) Springer Science+Business Media, LLC, part of Springer Nature 2020

Correction to: Journal of Medical Systems (2019) 43: 325. https://doi.org/10.1007/s10916-019-1457-4

In the original version of this article, the authors' units in the affiliation section are, unfortunately, incorrect. Jining No.1 people's hospital and Affiliated Hospital of Jining Medical University are two independent units and should not have been combined into one affiliation. The correct format of the authors' affiliations are shown in this article.

The online version of the original article can be found at https://doi.org/ 10.1007/s10916-019-1457-4

Xinhong Lu

hide0717@163.com

1 Affiliated Hospital of Jining Medical University,

Jining 272000, Shandong, China

2 Jining No.1 people's hospital, Jining 272000, Shandong, China 